\title{
In vitro cytocompatibility evaluation of chitosan/graphene oxide 3D scaffold composites designed for bone tissue engineering
}

\author{
Sorina Dinescu ${ }^{\mathrm{a}}$, Mariana Ionita ${ }^{\mathrm{b}}$, Andreea Madalina Pandele ${ }^{\mathrm{b}}$, Bianca Galateanu ${ }^{\mathrm{a}, \mathrm{c}}$, Horia $^{\mathrm{c}}$ \\ Iovu $^{\mathrm{b}}$, Aurel Ardelean ${ }^{\mathrm{c}}$, Marieta Costache ${ }^{\mathrm{a}}$ and Anca Hermenean ${ }^{\mathrm{c},}$ \\ ${ }^{a}$ Department of Biochemistry and Molecular Biology, University of Bucharest, 91-95 Splaiul \\ Independentei, 050095 Bucharest, Romania \\ ${ }^{b}$ University Politehnica of Bucharest, Calea Victoriei 149, 010072 Bucharest, Romania \\ ${ }^{c}$ Department of Experimental and Applied Biology, Institute of Life Sciences, Vasile Goldis Western \\ University of Arad, 86 Rebreanu, 310414 Arad, Romania
}

\begin{abstract}
Extensively studied nowadays, graphene oxide (GO) has a benefic effect on cell proliferation and differentiation, thus holding promise for bone tissue engineering (BTE) approaches. The aim of this study was not only to design a chitosan 3D scaffold improved with GO for optimal BTE, but also to analyze its physicochemical properties and to evaluate its cytocompatibility and ability to support cell metabolic activity and proliferation. Overall results show that the addition of GO in the scaffold's composition improved mechanical properties and pore formation and enhanced the bioactivity of the scaffold material for tissue engineering. The new developed CHT/GO $3 \mathrm{wt} \%$ scaffold could be a potential candidate for further in vitro and in vivo osteogenesis studies and BTE approaches.
\end{abstract}

Keywords: Chitosan, graphene oxide, 3D scaffold, biocompatibility, bone tissue engineering

\section{Introduction}

Since carbon-based nanomaterials showed versatility and good properties for biomedical applications, graphenes and graphene oxide (GO) have been extensively studied [1]. GO particularly holds great promise for tissue engineering applications, due to its unique physicochemical properties- hydrophilicity, high dispersibility and ability to promote biological interactions through the functional groups on its surface [2-4].

\footnotetext{
${ }^{*}$ Corresponding author: Anca Hermenean, Department of Experimental and Applied Biology, Institute of Life Sciences, Vasile Goldis Western University of Arad, 86 Rebreanu, 310414 Arad, Romania. Tel.:+40257282839; Fax: +40257282839; E-mail: anca.hermenean@gmail.com.
} 
GO was shown to influence positively the proliferation and differentiation of mesenchymal stem cells (MSCs) during in vitro culture [5,6]. Thus, it started to be used in association with other biocompatible materials in order to enhance their biological activity and to facilitate their interaction with cells [7-9]. Chitosan (CHT) was used in this study due to its good biocompatibility, biodegradability and wound-healing properties [10]. This naturally occurring polymer is especially attractive as a bone scaffold material because it supports the attachment and proliferation of osteoblast cells, as well as formation of mineralized bone matrix [11]. In this context, the aim of this study was to develop a novel engineered chitosan 3D scaffold enriched with GO in an optimal proportion to allow efficient cellsubstrate interaction, to analyze its physicochemical properties and to evaluate its cytocompatibility in terms of cytotoxicity and ability to support cell metabolic activity and proliferation.

Although bone is capable of regenerative growth and remodeling, bone tissue engineering (BTE) strategies are currently developing to restore bone function after disease, trauma or tumor resection $[12,13]$. An appropriate combination of scaffold properties and cells with potential for bone repair is required for efficient BTE approaches. Thus, novel scaffold formula improved with GO, designed for BTE, and a murine preosteoblast cell line were used in these experiments in order to investigate if the scaffold actively supports cells during their growth and development. Additionally, proving biocompatibility of these materials with murine preosteoblasts will allow further in vivo osteogenic differentiation studies on mouse models.

\section{Materials and methods}

\subsection{Materials}

Graphene oxide was supplied by National Institute for Research and Development in Microtechnologies (Romania), prepared according to Hummers procedure [14]. Acetic acid ( $\geq 99.7 \%)$ and chitosan from crab shells were purchased from Sigma-Aldrich. All materials were used without further purification and the water used in this work was double distilled water.

The cytotoxic potential exerted by the scaffolds indirectly on VERO cells was quantified using "In vitro toxicology assay kit lactate dehydrogenase based" (Sigma-Aldrich Co., Steinheim, Germany), following manufacturer's instructions. The amount of metabolically active mouse preosteoblast cells and their proliferation rate when cultivated in contact with the materials was quantitatively determined using MTT colorimetric assay (Sigma-Aldrich Co., Steinheim, Germany).

\subsection{Fabrication of $C H T / G O 3 D$ porous scaffolds}

$2.5 \mathrm{~g}$ of CHT were mixed with $250 \mathrm{ml}$ acetic acid solution $\left(10 \%\right.$ by weight in water) at $-50^{\circ} \mathrm{C}$ in order to form a homogeneous viscous solution. Further, different contents of graphene oxide $(0 ; 0.5$ and 3 weight percent (wt\%)) were added into CHT solution and mixed by ultrasonication for $1 \mathrm{~h}$ at room temperature. The homogeneous solutions were casted onto transparent glass Petri dish, then frozen overnight at $-70^{\circ} \mathrm{C}$ and freeze-dried for 2 days at $-50^{\circ} \mathrm{C}(0.040 \mathrm{mbar})$. After sublimation of ice crystals by freeze-drying, the polymer structure became porous. The $3 \mathrm{D}$ dried materials were thermal treated in vacuum, according to the following procedure: $50^{\circ} \mathrm{C}$ for $30 \mathrm{~min}, 70^{\circ} \mathrm{C}$ for $30 \mathrm{~min}$ and overnight at $90^{\circ} \mathrm{C}$. The obtained samples were then subjected to advanced characterization of the bulk surface and to in vitro biocompatibility assessment. 


\subsection{Physicochemical and morphological characterization of CHT/GO scaffolds}

Fourier transform infrared (FT-IR) spectra of CHT and CHT/GO 3D materials were recorded in 600 $\div 4000 \mathrm{~cm}^{-1}$ range with $4 \mathrm{~cm}^{-1}$ resolution on SHIMADZU 8900 equipment. The structural characteristics of the 3D materials were studied by X-ray diffraction (XRD) using Panalytical X'Pert Pro MPD instrument with $\mathrm{Cu} \mathrm{K} \alpha$ radiation. The morphology of CHT/GO 3D composites was studied using QUANTA INSPECT F scanning electron microscope (SEM) with $1.2 \mathrm{~nm}$ resolution and an energy dispersive X-ray spectrometer with a resolution of $133 \mathrm{eV}$ to $\mathrm{MnK} \alpha$. The samples were coated using the ion-beam sputter device with gold particles, previous to SEM analysis.

\subsection{In vitro biocompatibility assessment of $3 D$ CHT/GO materials}

\subsubsection{Cell culture model}

Direct biocompatibility studies, where the materials were put in direct contact with cells, were performed using the murine preosteoblasts cell line MC3T3-E1 (ECACC, code 99072810). The cells were cultivated in Minimum Essential Medium Eagle (MEM) alpha culture medium, supplemented with $2 \mathrm{mM}$ Glutamine and $10 \%$ Fetal Bovine Serum (FBS). The resulting 2D cell-material construct was maintained in standard culture conditions, at $37^{\circ} \mathrm{C}, 5 \% \mathrm{CO}_{2}$ and adequate humidity.

For indirect cytocompatibility studies, standardized cell line VERO from African green monkey kidney (88020401, Sigma) was used, according to ISO 10993-5:2009 recommendations. Cells were cultivated in DMEM medium supplemented with $2 \mathrm{mM}$ L-Glutamine and 10\% FBS until use.

\subsubsection{Indirect cytotoxicity studies}

For the purpose of indirect cytocompatibility tests, VERO cells were seeded in 24-well plates at an initial density of $10^{4}$ cells $/ \mathrm{cm}^{2}$. Cells were allowed to adhere to the substrate and were maintained in 1 $\mathrm{mL} /$ well of specific culture medium until they reached approximately $80 \%$ confluency.

In order to test the cytotoxic effect of CHT and CHT/GO composites upon the standardized VERO cell line, each scaffold composition was submersed and maintained in $3 \mathrm{~mL}$ of DMEM medium for 24 $\mathrm{h}$, in standard conditions of culture. The extracts obtained for each material composition were harvested and maintained at $37^{\circ} \mathrm{C}$.

After the cells had reached $80 \%$ confluency, the specific VERO culture medium was replaced with $1 \mathrm{~mL} /$ well of each of the harvested extracts. VERO culture was maintained in contact with these extracts for another $24 \mathrm{~h}$, in standard conditions of $37^{\circ} \mathrm{C}, 5 \% \mathrm{CO}_{2}$ and adequate humidity. Triplicate wells seeded with VERO cells were used for each scaffold composition extract. In order to evaluate the cytotoxic potential of the materials, an untreated bidimensional culture on a plastic surface was considered as a reference (2D control).

After this interval of $24 \mathrm{~h}$, lactate dehydrogenase (LDH) cytotoxicity assay was performed to provide information about potential cytotoxic effects of the analyzed materials. Before removing the extracts after $24 \mathrm{~h}$ of exposure to cell culture, $50 \mu \mathrm{L}$ of media were harvested and mixed with the kit components, following manufacturer's instructions. After 20 minutes of incubation at room temperature, the resulting solution was measured by means of spectrophotometry at $490 \mathrm{~nm}$ (Appliskan ThermoScientific).

The visualization of VERO cell morphology after 24-h exposure to scaffolds' extracts was revealed by phase-contrast microscopy, using 10x magnification, on an Olympus IX71 equipment. 


\subsubsection{Achievement of $3 D$ cultures}

3T3E1 mouse preosteoblasts were seeded on the surface of CHT, CHT/GO $0.5 \mathrm{wt} \%$ and CHT/GO 3 $\mathrm{wt} \%$ at an initial density of $1.8 \times 10^{5} \mathrm{cells} / \mathrm{cm}^{2}$. Cell suspension was allowed to diffuse through the pores of the scaffolds in order to obtain 3D cell-scaffold culture, further named as bioconstruct. The bioconstructs were incubated in specific culture medium for seven days at $37^{\circ} \mathrm{C}, 5 \% \mathrm{CO}_{2}$ and adequate humidity.

\subsubsection{MTT viability assay}

The MTT assay allows the evaluation of cell survival by reduction of a tetrazolium salt solutionMTT (3-(4,5-dimethylthiazolyl-2)-2,5-diphenyltetrazolium bromide) to insoluble purple formazan crystals by all metabolically active cells. The viability and proliferation rates of murine preosteoblasts in direct contact with CHT, CHT/GO $0.5 \mathrm{wt} \%$ and CHT/GO $3 \mathrm{wt} \%$ were determined using MTT spectrophotometric assay after two and seven days of culture. Culture media were removed and the cells were incubated with $1 \mathrm{mg} / \mathrm{mL}$ MTT solution for $4 \mathrm{~h}$, in standard culture conditions. The resulted formazan crystals were solubilized in isopropanol and the concentration of the solution was determined by spectrophotometry at $550 \mathrm{~nm}$ (Appliskan ThermoScientific).

\subsection{Statistical analysis}

The statistical evaluation of the data was done using one-way ANOVA method followed by Bonferroni multiple comparison test. The results were expressed as a mean \pm S.D. using GraphPad Prism Software, version 3.03 for Windows.

\section{Results and discussion}

\subsection{Physicochemical and morphological characterization of CHT/GO scaffolds}

The obtained CHT and CHT/GO composite materials presented a porous texture and compressibility and had a thickness of $0.8 \pm 0.2 \mathrm{~cm}$. At high magnification, the surface of CHT and CHT/GO composite 3D structures displayed a porous structure with open and well interconnected pores (Figure 1). However, an apparent difference between pure CHT and CHT/GO composite scaffolds was observed. The surface of pure CHT displayed a rough morphology with undefined pores (Figure 1A). Conversely, even for small GO amount added, the pores began to define, forming heterogeneous pore arrangements (Figure 1B). By further increasing the GO amount to $3 \mathrm{wt} \%$ in the composite material, the SEM images revealed a highly interconnected porosity and well defined pores (Figure 1C).
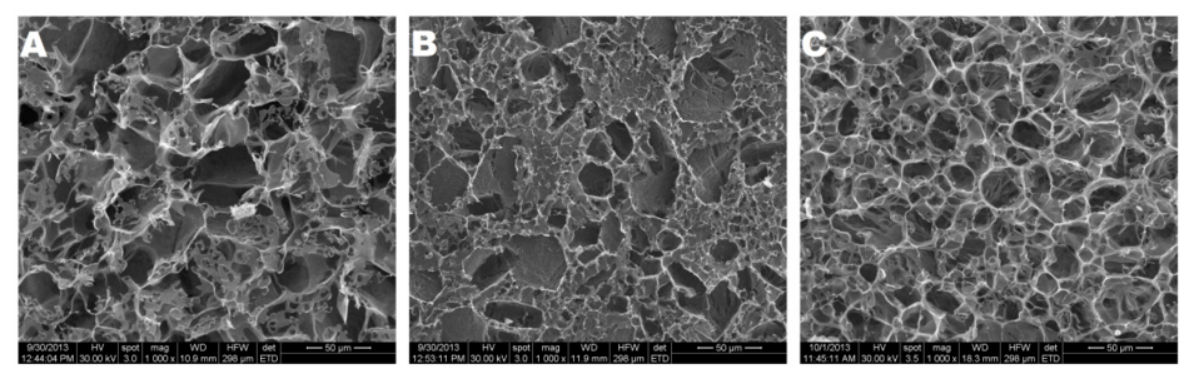

Fig. 1. SEM Images for A) CHT; B) CHT/GO $0.5 \mathrm{wt} \%$; C) CHT/GO $3 \mathrm{wt} \%$. 

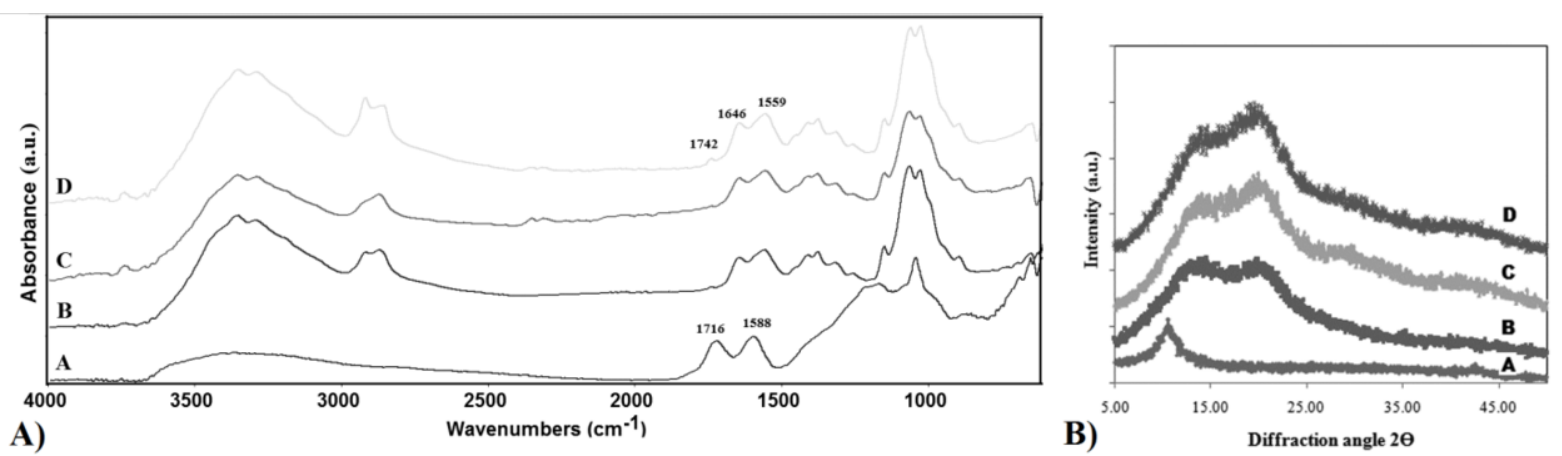

Fig. 2. GO (A); CHT (B); CHT/GO $0.5 \mathrm{wt} \%$ (C) and CHT/GO $3 \mathrm{wt} \%$ (D) spectra as revealed by A) FT-IR and B) XRD measurements.

Based on the SEM results, it was noticed that the addition of GO into the polymer matrix led to a decrease of interconnected pores size. The pore size of CHT ranged between 22 and $55 \mu \mathrm{m}$, while for the CHT/GO nanocomposites it varied from 4 to $30 \mu \mathrm{m}$.

The resulting massive porous structure is believed to be advantageous for cell attachment and proliferation allowing ingrowths of cells.

FT-IR measurements were performed to study the interaction between GO and CHT. Figure 2 exhibits the FT-IR spectra for GO, CHT and the CHT/GO composite materials with different GO loading. The GO spectrum (Figure 2A) revealed the presence of oxygen-containing functional groups. The two distinct peaks at $1716 \mathrm{~cm}^{-1}$ and $1588 \mathrm{~cm}^{-1}$ are assigned to $\mathrm{C}=\mathrm{O}$ stretching vibration of carboxylic group and $\mathrm{C}=\mathrm{C}$ stretching mode of the $\mathrm{sp}^{2}$ network, respectively. $\mathrm{CHT}$ spectrum displayed two characteristic bands at $1646 \mathrm{~cm}^{-1}$ and $1559 \mathrm{~cm}^{-1}$ attributed to amide I and III of $\mathrm{C}=\mathrm{O}$ stretching vibration and $\mathrm{N}-\mathrm{H}$ bending of $-\mathrm{NH}_{2}$. In comparison to CHT spectrum, the presence of the peak attributed to the carboxylic groups from GO surface was observed in the $\mathrm{CHT} / \mathrm{GO}$ composite materials spectra. This observation indicated GO presence in the composite materials. By increasing the GO amount, the peak was shifted to higher values $\left(1742 \mathrm{~cm}^{-1}\right)$ and became more intensive.

XRD measurements were performed to study the arrangement of polymer chains in the system. XRay diffractograms for GO, CHT and CHT/GO composites with different GO content ( 0.5 and $3 \mathrm{wt} \%$ ) measured at $25^{\circ} \mathrm{C}$, are presented in Figure $2 \mathrm{~B}$. A peak at $10.92^{\circ}$ was assigned to $\mathrm{GO}$, similar to the values previously reported in literature. XRD pattern of CHT exhibited two diffraction peaks at $12.76^{\circ}$ assigned to hydrated crystalline structure and a broad peak at $19.79^{\circ}$ which indicated the presence of an amorphous structure. The XRD pattern of CHT/GO enriched with $0.5 \mathrm{wt} \% \mathrm{GO}$ was nearly the same as for the pure CHT. The arrangement of the polymer chains seemed to be affected by the addition of GO in the CHT matrix. The peak at $19.79^{\circ}$ looked broad for the pure CHT spectrum, while CHT/GO composites with $0.5 \mathrm{wt} \%$ GO gave a slightly more intense peak. Moreover, when $3 \mathrm{wt} \%$ GO was loaded, the peak appeared sharper and more intense than for the composites with $0.5 \mathrm{wt} \%$, which indicated a more uniform arrangement of the polymer chains with GO additions. Furthermore, the diffraction peak of GO disappeared, which suggested a good exfoliation of GO within the polymer matrix. The more orderly arrangement of the polymer chains could be attributed to CHT adsorption on the exfoliated GO nanosheets. 


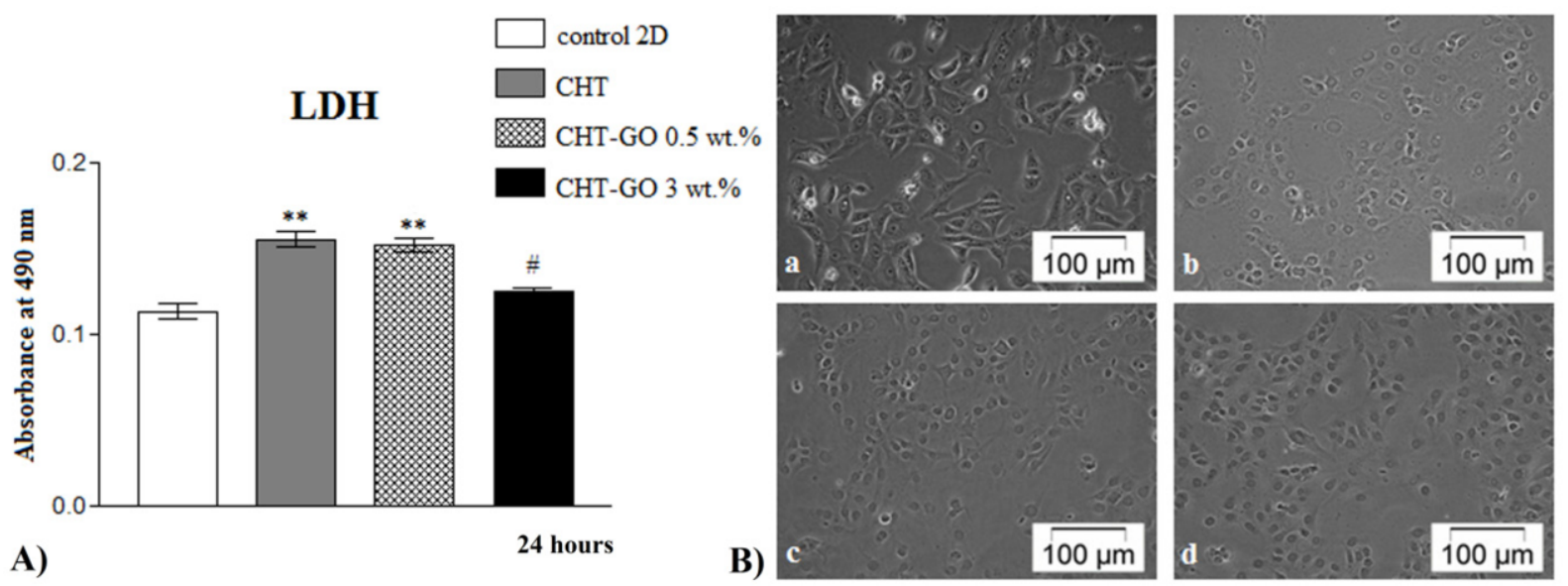

Fig. 3. (A) The cytotoxic potential of $\mathrm{CHT}$ and $\mathrm{CHT} / \mathrm{GO}$ composites extracts on cells after $24 \mathrm{~h}$ of culture, as revealed by LDH assay. Statistical significance: \#- $<<0.05$ (CHT/GO $3 \mathrm{wt} \%$ vs. CHT/GO $0.5 \mathrm{wt} \%$ ); **- $<<0.01$ (CHT/GO $3 \mathrm{wt} \% \mathrm{vs}$. CHT); (CHT/GO $0.5 \mathrm{wt} \%$ vs. CHT); (B) VERO cells morphology after $24 \mathrm{~h}$ of treatment with CHT (b), CHT/GO $0.5 \mathrm{wt} \%$ (c) and $\mathrm{CHT} / \mathrm{GO} 3 \mathrm{wt} \%$ (d) extracts, as compared to the normal, untreated VERO phenotype (a), as revealed by phase contrast microscopy.

\subsection{Indirect tests}

All tested CHT/GO composites and CHT control were evaluated for their cytotoxic potential indirectly, by assessing the effect of their extracts on VERO cells after 24 hours of culture. The results of LDH cytotoxicity assay (Figure 3A), proportional to the levels of LDH released in the medium by the non-viable cells with a damaged membrane, showed that all materials displayed low cytotoxicity. However, cells cultivated in the presence of pure CHT extract displayed the highest levels of LDH activity as compared to $2 \mathrm{D}$ control $(\mathrm{p}<0.01)$. A comparable cytotoxic potential was also registered for CHT/GO $0.5 \mathrm{wt} \%$, suggesting that the addition of $0.5 \mathrm{wt} \% \mathrm{GO}$ in scaffold's composition did not bring significant differences in terms of cytocompatibility. Remarkably, in the presence of CHT/GO $3 \mathrm{wt} \%$ extract, LDH activity was statistically significant lower than the ones registered for CHT and CHT/GO $0.5 \mathrm{wt} \%(\mathrm{p}<0.01)$, but comparable to the one in $2 \mathrm{D}$ control. This result suggests a possible influence of $3 \mathrm{wt} \% \mathrm{GO}$ content in the material upon cell behavior and metabolic activity.

The quantitative results of the LDH assay confirmed the observations resulted from the investigation of cell morphology by phase contrast microscopy (Figure 3B). Due to the 24-h interaction with the extracts, VERO cells displayed a modified phenotype as compared to the untreated culture control (Figure 3B-a). The most significant phenotypical changes were observed in cells exposed to CHT extract, while the most similar phenotype to control was displayed by cells cultivated in the presence of $\mathrm{CHT} / \mathrm{GO} 3 \mathrm{wt} \%$ extract.

\subsection{Biocompatibility of $C H T / G O$ materials in direct contact with mouse preosteoblasts}

When put in direct contact with CHT and CHT/GO composites, overall high percentage of the seeded cells remained metabolically active, suggesting a good biocompatibility of the materials. Mouse preosteoblasts proliferation in contact with these materials described an increasing trend during seven days of culture. Interestingly, cells cultivated in contact with CHT/GO 0.5 and $3 \mathrm{wt} \%$ registered a significantly $(\mathrm{p}<0.001)$ higher rate of viability and proliferation than the ones cultivated in contact with 


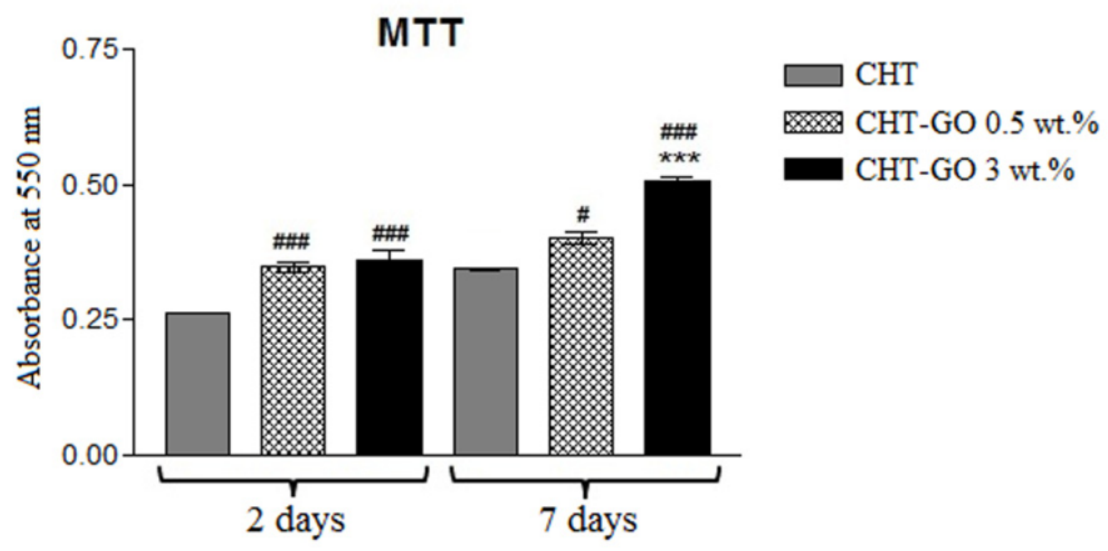

Fig. 4. The quantification of MC3T3-E1 preosteoblasts metabolic activity in contact with CHT and CHT/GO composites after 2 and 7 days of culture, as determined by MTT assay. Data analysis was based on mean \pm SD $(n=3)$. Statistical significance: \#- $<<0.05$ (CHT/GO $0.5 \mathrm{wt} \% 7$ days vs. CHT 7 days); \#\#\#- $<<0.001$ [(CHT/GO $0.5 \mathrm{wt} \% 2$ days vs. CHT 2 days); (CHT/GO 3 wt $\% 2$ days vs. CHT 2 days); (CHT/GO 3 wt\% 7 days vs. CHT 7 days)]; ***- p<0.001 (CHT/GO 3 wt $\% 7$ days vs. CHT/GO $0.5 \mathrm{wt} \% 7$ days).

CHT control after 2 days of culture. This difference in cell behavior could be attributed to the presence of GO, which could have a role in enhancing cells' metabolic activity and proliferation. The importance of GO content in the material's composition was also found to be crucial for cell activity, since after seven days of culture murine preosteoblasts proliferated more $(\mathrm{p}<0.001)$ in contact with 3 $\mathrm{wt} \% \mathrm{GO}$, than in the presence of only $0.5 \mathrm{wt} \% \mathrm{GO}$. Overall, both CHT/GO $0.5 \mathrm{wt} \%$ and CHT/GO 3 $\mathrm{wt} \%$ registered better biocompatibility, in terms of cell metabolic activity and proliferation, than CHT control after one week of culture, but with different rates $\mathrm{p}<0.05$ and $\mathrm{p}<0.001$, respectively.

\section{Conclusion}

The studied CHT-based 3D materials, enriched with GO in different proportions, appear to be ideally designed for bone tissue engineering applications in terms of biocompatibility and properties to promote and support cell growth and proliferation. The extracts released by these composites in the surrounding environment do not exhibit significant cytotoxic effects. The results suggest that the addition of GO in the scaffold's composition not only improves mechanical properties and pore formation, but also enhances the bioactivity of the scaffold material for tissue engineering. These effects on cell behavior appear to be proportional to the concentration of GO found in the formulation of the scaffold. In conclusion, $\mathrm{CHT} / \mathrm{GO} 3 \mathrm{wt} \%$ could be a potential candidate for further in vitro and in vivo osteogenesis studies and bone repair approaches.

\section{Acknowledgement}

This work was supported by the strategic grant POSDRU/159/1.5/S/133391, Project "Doctoral and Post-doctoral programs of excellence for highly qualified human resources training for research in the field of Life sciences, Environment and Earth Science" co-financed by the European Social Fund 
within the Sectorial Operational Program Human Resources Development 2007-2013 and by the Romanian CNCS-UEFISCDI Research Project (Grant No. PCCA140/2012).

\section{References}

[1] O.N. Ruiz, K.A.S. Fernando, B. Wang, N.A. Brown, P.G. Luo, N.D. McNamara, M. Vangsness, Y. Sun and C.E. Bunker, Graphene oxide: A nonspecific enhancer of cellular growth, ACS Nano. 5 (2011), 8100.

[2] D.R. Dreyer, S. Park, C.W. Bielawski and R.S. Ruoff, The chemistry of graphene oxide, Chem. Soc. Rev. 39 (2010), 228.

[3] Y. Zhu, S. Murali, W. Cai, X. Li, J.W. Suk, J.R. Potts and R.S. Ruoff, Graphene and graphene oxide: Synthesis, properties, and applications, Adv. Mater. 22 (2010), 3906.

[4] M. Tang, Q. Song, N. Li, Z. Jiang, R. Huang and G. Cheng, Enhancement of electrical signaling in neural networks on graphene films, Biomaterials 34 (2013), 6402.

[5] G.-Y. Chen, D.W.-P. Pang, S.-M. Hwang, H.-Y. Tuan and Y.-C. Hu, A graphene-based platform for induced pluripotent stem cells culture and differentiation, Biomaterials 33 (2012), 418.

[6] T.R. Nayak, H. Andersen, V.S. Makam, C. Khaw, S. Bae, X. Xu, P.R. Ee, J. Ahn, B.H. Hong, G. Pastorin and B. Ozyilmaz, Graphene for controlled and accelerated osteogenic differentiation of human mesenchymal stem cells, ACS Nano. 5 (2011), 4670.

[7] E. Nishida, H. Miyaji, H. Takita, I. Kanayama, M. Tsuji, T. Akasaka, T. Sugaya, R. Sakagami and M. Kawanami, Graphene oxide coating facilitates the bioactivity of scaffold material for tissue engineering, Japanese Journal of Applied Physics 53 (2014), 06JD04.

[8] A.M. Pandele, S. Dinescu, M. Costache, E. Vasile, C. Obreja, H. Iovu and M. Ionita, Preparation and in vitro, bulk, and surface investigation of chitosan/graphene oxide composite films, Polymer Composites 34 (2013), 2116-2124.

[9] A.M. Pandele, M. Ionita, L. Crica, S. Dinescu, M. Costache and H. Iovu, Synthesis, characterization, and in vitro studies of graphene oxide/chitosan-polyvinyl alcohol films, Carbohydrate Polymers 102 (2014), 813-820.

[10] B. Guo, L. Glavas and A.C. Albertsson, Biodegradable and electrically conducting polymers for biomedical applications, Progress in Polymer Science 38 (2013), 1263-1286.

[11] S.K. Lan Levengooda and M. Zhang, Chitosan-based scaffolds for bone tissue engineering, J. Mater. Chem. B 2 (2014), 3161-3184.

[12] P. Yang, X. Huang, C. Wang, X. Dang and K. Wang, Repair of bone defects using a new biomimetic construction fabricated by adipose-derived stem cells, collagen I, and porous beta-tricalcium phosphate scaffolds, Experimental Biology and Medicine 238 (2013), 1331.

[13] M. Yang, N. Mandal, Y. Shuai, G. Zhou, S. Min and L. Zhu, Mineralization and biocompatibility of antheraea pernyi (A. pernyi) silk sericin film for potential bone tissue engineering, BioMed. Mater. Eng. 24 (2014), 815-824.

[14] W. S. Hummers Jr, R. E. Offeman, Preparation of Graphitic Oxide, American Chemical Society 80 (1958), 1339. 